Meta

Journal des traducteurs

Translators' Journal

\title{
Le traitement de la terminologie géographique, opération clef de l'aménagement toponymique au Québec
}

\section{Jean-Yves Dugas}

Volume 36, numéro 1, mars 1991

La terminologie dans le monde : orientations et recherches

URI : https://id.erudit.org/iderudit/002669ar

DOI : https://doi.org/10.7202/002669ar

Aller au sommaire du numéro

Éditeur(s)

Les Presses de l'Université de Montréal

ISSN

0026-0452 (imprimé)

Découvrir la revue

Citer cet article

Dugas, J.-Y. (1991). Le traitement de la terminologie géographique, opération clef de l'aménagement toponymique au Québec. Meta, 36(1), 218-224.

https://doi.org/10.7202/002669ar d'utilisation que vous pouvez consulter en ligne. 


\title{
LE, TRAITEMENT DE LA TERMINOLOGIE GÉOGRAPHIQUE, OPÉRATION CLEF DE L'AMÉNAGEMENT TOPONYMIQUE AU QUÉBEC
}

\author{
JEAN-YVES DUGAS \\ Commission de toponymie, Québec, Canada
}

La rivière peut être à sec, elle garde son nom

Proverbe africain

À première vue, il peut paraître délicat, voire risqué, de prospecter les rapports, ou encore les liens d'interdépendance que peuvent entretenir deux champs d'activité aussi peu compatibles, de prime abord, que la toponymie et la terminologie. L'une voit ses débuts remonter presque au paradis terrestre alors qu'Adam donne un nom aux animaux, pavant ainsi la voie à tous les dominants qui allaient reprendre le flambeau de ce donneur de noms, l'autre qui porte bien la jeune vingtaine et dont maints aspects font encore l'objet de recherche ou d'expérimentation. Alors que la terminologie a pour rôle d'instaurer un contrôle rigoureux du langage, la toponymie préside aux destinées d'un domaine où l'usage règne en maître avec ses formes parallèles, ses réduplications, ses approximations définitoires. La confrontation de ces deux disciplines relève davantage du combat qui met aux prises l'instinct et l'intellect, le cour et la raison, la spontanéité et le choix longuement réfléchi. Enfin, quoique la terminologie recrute des adeptes de plus en plus nombreux, elle demeure cependant la chasse gardée d'un groupe assez restreint de spécialistes, alors que la toponymie rejoint chacun d'entre nous dans la vie de tous les jours: nom de rues, adresses postales, parcours des transports publics, nouvelles de la radio et de la télévision, prévisions météorologiques, itinéraires de voyage, etc.

Il ne faut cependant pas croire qu'il faille abandonner le trésor toponymique québécois aux aléas d'une croissance anarchique, souvent fort problématique, car même dans un univers aussi peu réglementé que celui de l'art, les penseurs ont toujours estimé quc des balises, un certain encadrement, une structure se révélaient nécessaires. Le célèbre romancier français André Gide a traduit dans une percutante formule cette élémentaire nécessité : «L'art naît de contrainte, vit de lutte, meurt de liberté» (Gide : 17). Toute proportion gardée, cette loi de l'esprit s'applique à un domaine qui rejoint le grand public comme la toponymie.

Dans un esprit de sagesse et de réalisme, le législateur d'ici a voulu fixer un cadre général à l'intérieur duquel les noms de lieux puissent s'épanouir harmonieusement. À cet égard, on peut identifier deux aspects importants qui ont présidé à son action. En premier lieu, le fait que la Commission de toponymie ait été créée dans le cadre de la Charte de la langue française et rattachée administrativement à l'Office de la langue française apparaît comme singulièrement éloquent. On peut déceler dans cette action le désir du Gouvernement de l'époque de voir le travail de la Commission s'inscrire dans le cadre général d'une politique de francisation, tout en laissant à ses dirigeants le soin 
d'établir les différentes modalités d'une telle politique, dans le respect des divers particularismes toponymiques de la Belle Province.

Le second volet de l'intention du législateur demeure on ne peut plus manifeste. En effet, le paragraphe $c$ de l'article 125 de la Charte impose comme devoir à la Commission de toponymie d' «établir et normaliser la terminologie géographique, en collaboration avec l'Office». Ainsi, l'action normalisatrice de la Commission se voyait précisée et restreinte, dans la mesure où, d'une part, elle devait s'exercer dans un champ déterminé de la toponymie, la terminologie géographique, c'est-à-dire l'ensemble des termes ou expressions qui indiquent la nature d'un lieu nommé ou susceptible de l'être et, d'autre part, la normalisation de ces unités lexicales devait être effectuée de concert avec l'Office de la langue française, autorité de première instance en matière de terminologie au Québec.

$\mathrm{Si}$, de cette manière, l'esprit de la normalisation terminologique en toponymie était arrêté, il n'en allait pas ainsi pour autant de la lettre. En conséquence, après avoir brossé succinctement la toile de fond historique de la toponymie et de la terminologie géographique, nous exposerons, dans ses grandes lignes, la politique terminologique arrêté par la Commission de toponymie. Nous en détaillerons les modalités d'application en regard du substrat toponymique à l'aide d'une série d'illustrations concrètes qui permettront de mieux saisir la délicate situation qui préside aux contacts entre l'onomastique et la langue générale, laquelle doit loger à l'enseigne de la rigueur et de l'authenticité.

\section{UN PASSÉ... RÉCENT}

Quoique 1'histoire toponymique du Québec débute véritablement avec l'arrivée de la première vague colonisatrice du XVII siècle, précédée d'une période impossible à fixer avec précision dans le temps au cours de laquelle les Amérindiens ont baptisé généreusement leurs territoires de chasse et de migration, son encadrement administratif remonte à moins de 80 ans. En effet, instituée par un arrêté en conseil en date du 15 novembre 1912, la Commission de géographie du Québec était officiellement créée par une loi sanctionnée le 14 février 1920. Cet organisme relevait du ministre des Terres et Forêts et son mandat portait, entre autres, sur la dénomination des lieux, l'élimination de l'homonymie, l'uniformisation de la toponymie et des règles d'écriture des noms de lieux. Disposant de peu de personnel, l'organisme, qui n'en a pas moins accompli un travail méritoire, notamment quant à l'accroissement du caractère français de la toponymie québécoise, n'a pu jouer un rôle véritable dans l'aménagement de la toponymie en raison de ses pouvoirs restreints et d'un mandat étendu et peu explicite.

L'atteinte des objectifs de la Commission de géographie du Québec se trouvant souvent freinée par les limites de sa compétence en matière de noms géographiques, le Gouvernement devait, le 26 août 1977, créer la Commission de toponymie, laquelle prenait la relève de la Commission de géographie. La Charte de la langue française confiait à cet organisme des devoirs et des pouvoirs singulièrement élargis. Ceux-ci concernent essentiellement la détermination des critères de choix et des règles d'écriture des noms de lieux, l'attribution de dénominations aux endroits innommés, la diffusion de la nomenclature géographique officielle du Québec, le rôle d'organisme-conseil auprès du Gouvernement et des autres organismes de l'Administration, ainsi qu'un droit de regard et d'avis quant à la toponymie figurant dans les textes de l'Administration, dans la signalisation routière, l'affichage public et le matériel pédagogique approuvé par le ministère de l'Éducation. En outre, la Charte confiait à la Commission de toponymie un mandat spécifique pour établir et normaliser la terminologie géographique, en collaboration avec l'Office de la langue française (Québec, province, 1989). 
Depuis une dizaine d'années, des travaux de nature terminologique sont menés à la Commission de toponymie afin de satisfaire à ce devoir. À l'automne de 1979, une Commission de terminologie géographique était créée avec pour mandat, dans un premier temps, de traiter des termes génériques qui entrent dans la composition des odonymes ainsi que des types d'entités géographiques auxquelles ils sont attribués. À partir de 1982 jusqu'en 1985, un Comité de terminologie géographique a procédé à l'analyse et au traitement terminologique de termes géographiques ressortissant à des secteurs précis comme la morphologie littorale, l'hydrographie, la topographie, les lieux habités, etc. Ces travaux ont permis, à ce jour, de procéder à la normalisation de 113 termes et expressions géographiques, alors qu'un avis de recommandation était publié à la Gazette officielle du Québec pour 127 autres unités lexicales ${ }^{1}$.

\section{UNE POLITIQUE TERMINOLOGIQUE SOUPLE}

Afin de se doter d'un cadre général de traitement terminologique rigoureux et d'assurer la plus grande qualité possible à ses décisions, tout en ne dénaturant pas le caractère spontané du corpus toponymique québécois, la Commission de toponymie a établi certains principes qui traduisent la continuité de sa pensée et de son action.

Tout d'abord, la Commission limite l'exercice de son mandat à la terminologie associée directement à la toponymie, c'est-à-dire aux termes et aux expressions qui entrent dans la composition des noms de lieux qu'elle a, par ailleurs, le devoir d'officialiser, ou encore qui concernent de près ou de loin des toponymes. Ce choix témoigne du désir légitime d'éclairer et de garantir la qualité des actes d'officialisation qu'elle pose régulièrement.

Le respect de l'usage courant, mis de l'avant par les Conférences des Nations Unies sur la normalisation des noms géographiques ${ }^{2}$, auquel la Commission souscrit entièrement, constitue la pierre angulaire du traitement toponymique dont les conséquences terminologiques sont également prises en compte. La reconnaissance de l'apport du français québécois dans la toponymie guide aussi l'action de la Commission dans la mesure où les québécismes véhiculent les traits distinctifs de notre société.

Dans son action normalisatrice, la Commission de toponymie établit une nette distinction entre le générique et le spécifique d'un toponyme, de même qu'elle considère à part le type d'entité ${ }^{3}$ identifié par un nom de lieu. Le générique consiste en un élément du toponyme qui identifie de manière générale la nature de l'entité géographique dénommée (Embranchement Germain; Étang des Pissenlits), alors que le spécifique est l'élément du nom de lieu qui identifie de façon particulière l'entité géographique dénommée (Lac des Deux Iles; Sentier de la Promenade-des-Sours). Quant au type d'entité, il précise la variété d'accident géographique auquel se rattache le nom de lieu [Baie de l'Est (Témiscamingue)], où le type d'entité est baie; Rivière Maheu (Beauce), où le type d'entité est ruisseau.

Dans cette optique, l'intervention normalisatrice de la Commission est adaptée à la nature même de la catégorie des toponymes concernés. Ainsi, pour caractériser les entités administratives, les termes utilisés doivent être conformes aux définitions reconnues, notamment pour les voies de communication et pour les éléments du sous-sol ou de la surface du sol construits ou profondément modifiés par l'homme (digue, pont, réservoir). En matière d'entités naturelles (lac, mer, vallée), la conformité aux définitions reconnues peut céder le pas aux impératifs de l'usage qui s'en écarte parfois.

Le niveau de normalisation poursuivi est fonction également du secteur d'intervention. Ainsi, le vocabulaire mis à contribution pour caractériser les types d'entités géographiques fait l'objet d'une normalisation terminologique rigoureuse, alors que la conformité des termes génériques aux définitions normalisées demeure une règle 
beaucoup moins absolue. Les termes et expressions qui entrent dans la composition des spécifiques ne font pas l'objet d'une intervention normalisatrice, à l'exception de l'application des règles qui régissent la correction grammaticale.

\section{QUELQUES PARTICULARISMES DE LA TOPONYMIE QUÉBÉCOISE ET INCIDENCE TERMINOLOGIQUE}

Comme on a pu le constater jusqu'à présent, la problématique terminologique en géographie et en toponymie doit tenir compte du contexte québécois, de la proximité que le langage de la toponymie entretient avec la langue générale, du souci de sauvegarder l'originalité d'une partie du patrimoine culturel d'ici véhiculé par la toponymie. Voyons à l'aide d'exemples concrets les principaux éléments qui interviennent dans la démarche terminologique mise de l'avant et que l'on doit prendre en considération si l'on veut préserver le caractère authentique de nos noms de lieux.

\section{L'USAGE POPULAIRE}

Considéré comme la clef de voûte du traitement toponymique (voir supra), l'usage populaire en ce qu'il ne rationalise pas l'utilisation du langage et en raison de la multiplicité des intervenants contribue à enraciner dans le temps des dénominations qui apparaissent «inexactes» après coup en ce qu'elles ne concordent pas avec le vocabulaire terminologique rigoureusement établi.

À titre d'exemples, les terres à Proudfoot (Champlain), la rivière à Renaud (Charlevoix), la baie du Père-Tellier (Berthier) et le chenal à Saint-Denis (Papineau) identifient respectivement des champs, un ruisseau, un étang et une passe. Substituer au générique inadéquat le terme correct ferait preuve d'un interventionnisme envahissant qui risquerait de dénaturer à tout jamais l'héritage toponymique que nous ont légué nos ancêtres. C'est ici que la distinction établie entre le terme générique et le type d'entité qui accompagne chaque toponyme officiel figurant au Répertoire toponymique du Québec ou à la Gazette officielle prend toute sa valeur. Elle permet de respecter, dans la mesure du possible, les noms bien attestés par l'usage populaire et de fournir à l'utilisateur l'objet géographique défini selon des critères terminologiques en accord avec le vocabulaire géographique reconnu.

\section{LES RÉGIONALISMES}

On retrouve, en toponymie québécoise, un certain nombre de régionalismes en guise de génériques, tels barachois (Barachois à Montpetit au Saguenay), cabouron (Cabouron de la Barlette dans le Bas-Saint-Laurent), caye (Caye à Ti-Joseph au Saguenay), coulée (Coulée de la Coop au Saguenay), marche (Marche à Jérôme dans Labelle), plée (Grande plée Bleue à Lévis), crique (Crique Paquet en Abitibi) ${ }^{4}$. Un examen terminologique rapide du terme barachois permet de saisir la multiplicité des sens auxquels il a donné lieu : tantôt il recouvre la notion de barre de sable ou de gravier formée à l'embouchure d'une rivière dans les régions de marée, tantôt celle de petite anse ou havre, lieu de refuge. Parfois il désigne une étendue d'eau plus profonde séparée de la mer par un banc de sable et entourée de prairies naturelles, en d'autres occasions c'est à l'étang qu'on recourt en guise de terme équivalent ou substitutif. Le traitement terminologique de ce terme a permis d'établir que le type d'entité auquel il correspond généralement est lagune, et dans certains cas, anse. Il en va de même pour caye qui équivaut à rocher, gaine à anse, marche à marais ou marécage, plée à marais, crique à ruisseau, etc., ces régionalismes demeurant sauvegardés à titre d'éléments génériques. 


\section{LA VARIABILITÉ RÉGIONALE}

À ce phénomène se greffe celui qui consiste en ce que l'on pourrait appeler la variabilité sémantique de divers termes génériques d'une région à l'autre du Québec. Ainsi, la coulée à Dominique identifie un ruisseau du Saguenay, alors que la coulée à Dominique (sic) désigne un ravin en Gaspésie. Qui plus est, dans la même région, le Saguenay, la coulée Père-Babel dénomme un ruisseau alors que la coulée de la Coop constitue la dénomination d'un ravin! Il importe donc que l'examen de la notion de coulée permette de déterminer objectivement le type d'entité à lui être adjoint. Dans la majorité des cas, il est apparu que le terme ravin, au sens d'entaille profonde et étroite sur un versant, creusée par les eaux de ruissellement (Commission de toponymie: 24), convenait la plupart du temps, même si dans certains cas le ruisseau qui coule au fond du ravin s'est vu identifié comme une coulée.

\section{LE CONTEXTE NORD-AMÉRICAIN}

Étant donné que les noms de lieux meublent le paysage géographique particulier d'un peuple spécifique, ils reçoivent, d'une certaine façon, l'influence du contexte plus ou moins étendu dans lequel ils s’inscrivent. À cet égard, la situation du Québec, au sein d'une fédération où la culture anglaise domine et à proximité des États-Unis dont la force d'attraction puissante se révèle omniprésente, n'a pas manqué d'entraîner une certaine répercussion en ce qui a trait en particulier à la terminologie odonymique d'ici. Deux exemples spécifiques nous permettront de mieux saisir le phénomène.

Afin de tenir compte de la structure particulière et largement répandue chez nos voisins du Sud suivant laquelle des avenues généralement orientées dans la direction nord-sud coupent des rues généralement orientées dans la direction est-ouest, un avis de normalisation en a permis l'utilisation au Québec. Ainsi, un second sens a été ajouté à celui qui recouvre la notion de base de rue et d'avenue ${ }^{5}$, lequel rend compte de la réalité baptisée plan en damier.

Mềme si l'on reconnaît généralement que le générique d'un odonyme n'entretient pas de rapport obligé avec la forme qu'épouse la voie qui porte ce nom, croissant demeure une exception qui se veut la reconnaissance d'un phénomène anglo-américain, adapté et passé dans l'usage québécois. Bien que du point de vue linguistique ce terme provienne de l'anglais crescent et constitue en quelque sorte un anglicisme, il trouve sa justification à la fois sur un plan toponymique et sur un plan topographique. En effet, il identifie de façon fort précise une réalité que le terme rue ne suffit pas à rendre parfaitement, soit une voie en forme de demi-cercle. De plus, il permet de pallier un certain nombre de problèmes d'homonymie et contribue à enrichir l'odonymie québécoise, eu égard au très grand nombre de voies qui comportent le générique rue. Ces illustrations démontrent éloquemment le contexte spécial dans lequel évolue la toponymie québécoise et la nécessité pour l'organisme qui gère ce patrimoine de tenir compte de facteurs socio-géographico-culturels dans l'établissement de la terminologie géographique, sous peine de se couper entièrement de la réalité ou d'adopter une attitude malthusienne.

\section{LE POIDS DE L'HISTOIRE}

En odonymie rurale, le recours à des génériques comme chemin, côte, montée, route et rang suscite de redoutables embûches pour le terminologue. En effet, ces termes passablement anciens se sont chargés au fíl des ans d'un imposant et significatif bagage sémantique, rendant délicate la tâche de bien cerner la ou les notions qu'ils recouvrent. Comme l'intervention terminologique ne comporte pas d'effet rétroactif, la normalisation de leur définition tient compte de leur sens actuel, même si pour l'établir il a été 
nécessaire d'effectuer une recherche historique circonstanciée. Toutefois, certains génériques doivent à l'«histoire» leur survivance dans la toponymie officielle actuelle.

Il ne faudra pas s'étonner de retrouver en milieu urbain un chemin Sainte-Foy (Sainte-Foy), une Grande Allée (Québec) qui n'a plus d'allée que la dénomination, une route de la Suète (Sainte-Foy) en pleine ville, même si les voies concernées ne répondent plus à la nature même d'un chemin, d'une allée ou d'une route. Le tissu urbain s'étant progressivement développé et ayant insensiblement transformé le milieu desservi par ces voies de communication, les appellations ont quand même subsisté, se vidant en quelque sorte de leur contenu sémantique au profit d'un strict rôle de désignants. Ce phénomène de désémantisation - les usagers ne pensent plus à la nature d'un chemin, d'une allée ou d'une route - témoigne de la tradition historique. Si ces formes, survivances du passé, subsistent encore à bon escient, on ne saurait y recourir pour une dénomination actuelle ou à venir.

Le nombre de problèmes terminologiques soulevés par la toponymie québécoise, et dont un mince échantillon a été exposé plus avant, démontre éloquemment la complexité que revêt l'intervention normalisatrice de la Commission de toponymie. En outre, cette action ne saurait s'effectuer adéquatement sans tenir rigoureusement compte de différentes données dont l'usage, les particularismes toponymiques locaux, le contexte linguistique, lesquelles demeurent des facteurs fondamentaux et majeurs de l'aménagement de la toponymie au Québec.

La réalité géographique qui relève strictement du milieu québécois ne peut, de toute évidence, être exprimée par des termes qui témoignent d'une autre civilisation et d'un autre usage, sous peine de dénaturer entièrement le patrimoine local. Notre insertion dans le continent nord-américain ne peut être passée sous silence lors de prise de décision à caractère linguistique ou terminologique en toponymie. D'un autre côté, il faut aussi prendre en considération la nécessité d'être compris - en cela la Commission doit assumer un rôle de premier plan - et le recours généreux à des régionalismes, s'il veut combler un légitime besoin d'authenticité, ne doit en aucun cas priver la collectivité internationale d'un droit tout aussi légitime à la saisie des termes utilisés. Cette attitude paraît d'autant plus indiquée dans un domaine de «propriété» générale comme la toponymie.

De plus, il convient de songer au rôle de nommant de la Commission qui, si elle recueille la plupart des toponymes qu'elle sanctionne, n'en assume pas moins la responsabilité de dénommer maints endroits ou entités, aux termes mêmes des pouvoirs que la Loi lui a confiés. L'organisme puise donc abondamment dans le matériel terminologique géographique mis au point lorsqu'elle soumet la nomenclature toponymique au creuset épurateur de la normalisation ou de l'aménagement, jouant ainsi son rôle de manière efficace et éclairée.

L'interventionnisme dans le domaine de la toponymie, s'il satisfait d'abord aux exigences de la Charte de la langue française, permet d'harmoniser et de préciser la teneur de cette importante facette du patrimoine culturel. La démarche terminologique constitue un élément rationnel important qui préside à l'élaboration et à l'utilisation des noms de lieux d'ici. Même s'il s'agit d'un domaine au sein duquel les forces créatrices impressionnistes et spontanées jouent un rôle plus primordial que dans tout autre, la terminologie permet de concilier ces dernières avec celles non moins importantes de l'entendement, en un harmonieux équilibre entre la rigueur dénominative et l'authenticité culturelle. 


\section{NOTES}

1. L'ensemble des termes et expressions géographiques normalisés ou recommandés par la Commission de toponymie et l'Office de la langue française figurent dans Commission de toponymie (1988), accompagnés de leurs définitions.

2. Par ailleurs, lors de la Première Conférence des Nations Unies sur la normalisation des noms géographiques tenue en 1967, la recommandation 19 suggérait «d'entreprendre une étude sur la nature des entités géographiques qui portent un nom dans une région donnée (...). Ces études pourront mettre en évidence des faits importants qui permettront de mieux comprendre ce que sont les entités géographiques désignées. Elles pourront aussi faire apparaitre les insuffisances des dictionnaires à cet égard.» (Nations Unies: 5).

3. Cette nette distinction se traduit concrètement, dans le Répertoire toponymique officiel, par la présence d'une colonne type d'entité qui suit immédiatement l'entrée toponymique.

4. Ces quelques exemples ne constituent, bien évidemment, qu'une modeste partie des régionalismes géographiques québécois qui ont pénétré en toponymie. La même démonstration pourrait être reprise à propos de batture, button, décharge, morne, platon, savane, etc.

5. Les termes avenue et rue présentent les sens normalisés suivants: «avenue, n. f. $1^{\circ}$ Voie de communication urbaine plus large que les rues, desservant un quartier ou une partie d'une ville, ou pouvant conduire à un lieu bien identifié. $2^{\circ}$ Dans un système de dénomination basé sur l'orientation des voies de circulation (plan en damier), voie urbaine située dans un axe perpendiculaire à celui des voies portant le nom de rue.» (Gazette officielle du Québec, 8 décembre 1980, p. 11 826);

«rue, n. f. $1^{\circ}$ Voie de communication généralement bordée de bâtiments dans une agglomération. $2^{\circ}$ Dans un système de dénomination basé sur l'orientation des voies de communication (plan en damier), voie située dans un axe perpendiculaire à celui des voies portant le nom d'avenue.» (Ibid.).

\section{BIBLIOGRAPHIE}

COMMISSION DE TOPONYMIE (1988) : Terminologie géographique de la toponymie québécoise, Québec, Commission de toponymie, Document de travail.

GIDE, André (1947): «L'évolution du théâtre», Nouveaux prétextes, Paris, Mercure de France, pp. 11-27.

HAMELIN, Louis-Edmond (1989): «Aspects terminologiques de la toponymie», Actes du Stage international de formation en toponymie, Québec, 7-19 août 1988, Québec, Commission de toponymie, pp. 371-382.

MORISSONNEAU, Christian et Jean-Marc NICOLE (1972): «La terminologie géographique archaïque et dialectale dans les noms de lieux du Québec», Cahiers de géographie du Québec, 16-38, Québec, pp. 325-334.

NATIONS UNIES (1968): Conférence des Nations Unies sur la normalisation des noms géographiques, Genève, 4-22 septembre 1967, 1, Rapport de la Conférence, New York, Nations Unies.

POIRIER, Jean (1979): «Terminologie et choronymie», Actes du $\sigma^{e}$ Colloque international de terminologie, Pointe-au-Pic (Québec), 2-6 octobre 1977, Québec, Office de la langue française, pp. 259-271.

QUÉBEC, Province (1987): Répertoire toponymique du Québec 1987, Québec, Les Publications du Québec.

QUÉBEC, Province (1989): Charte de la langue française, Québec, Éditeur officiel du Québec. 